\title{
Postponed Fracture of Femur Treated by Novel “Fixation in situ” Approach with llizarov: A Case Report
}

\author{
Ranjit Kumar Baruah $^{1}\left[\right.$ D. Shyam Sunder Sivaraman ${ }^{1}[\mathbb{C}$
}

Received: 1 July 2020 / Accepted: 28 August 2020 / Published online: 16 September 2020

(c) Indian Orthopaedics Association 2020

\begin{abstract}
The term "Postponed Fractures" (PF) appears to be more logical when one talks about neglected fractures. The "Fixation in situ" (FIS) approach with Ilizarov is a novel concept to treat such case. A 35-year male with comminuted subtrochanteric fracture with a big butterfly fragment (left femur) reported after 3 weeks of injury. He was ready for surgery at 6 weeks of injury with $5 \mathrm{~cm}$ shortening and external rotation deformity. Apprehending that Open surgery at that time would invite extensive soft tissue dissection resulting in infection, "Fixation in situ" approach was contemplated. A Hybrid Ilizarov Femoral frame was mounted without disturbing the fracture. Corticotomy through distal metaphysis was done to correct rotation and shortening. Accordion manoeuvre (AM) was also applied. The apparatus was removed at 6 months. He regained length, rotation and full function.
\end{abstract}

Keywords Postponed fractures $\cdot$ Ilizarov $\cdot$ Fixation in situ

\section{Introduction}

When the management of acute fractures is delayed for various reasons, the term Neglected fractures is commonly used. There is no defined period of delay to coin such a fracture as "neglected," [1]. The term "Postponed Fractures" (PF) seems to be logical in such cases that keeps aside the derogatory term "Neglect" that has extreme connotations. In postponed fractures, because of delay, proper management according to standard protocol for fresh fractures cannot be offered and, hence, a newer concept has emerged called "Fixation in situ" (FIS). Although Ilizarov concept and technology have revolutionized the Orthopaedic treatment, there is no mention of FIS concept in the available English literature. We have treated a PF of diaphysis of femur by this concept using Ilizarov technology yielding satisfactory results. We intend to report the case in terms of strategies used.

Ranjit Kumar Baruah rkb7887@gmail.com

Shyam Sunder Sivaraman shyam.arun90@gmail.com

1 Department of Orthopaedics, Assam Medical College Hospital, Dibrugarh, Assam, India

\section{Materials and Methods}

Case: GA, a 35-year male sustained comminuted subtrochanteric fracture with a big butterfly fragment left femur after being hit by a vehicle. He reported to us after 3 weeks of injury without any preliminary medical treatment. He was put on skin traction with Thomas Splint and necessary investigations were done. By the time he was ready for surgery, the fracture became 6 weeks old. There was shortening of $5 \mathrm{~cm}$ accompanied by 15 degrees external rotation deformity. He did not have any co-morbid conditions. At this point, the pain became less and he could lift the limb actively with some effort and X-ray showed visible callus. With this clinical background, we thought that with continuation of external immobilization, his fracture would proceed to heal but the rotational deformity and shortening would require some surgery. Standard open reduction and internal fixation would require extensive soft tissue dissection, thereby denuding the fragment and resultant avascularity would lead to infection.

We proposed to accept the displacement and correct the rotation and shortening by corticotomy. Accordingly, A Hybrid Ilizarov Femoral frame was mounted. Corticotomy through distal metaphysis was done and de-rotation was accomplished on table. Distraction of $1 \mathrm{~mm}$ was started after a latency of 7 days and continued till limb length was regained. Ring was further kept for another 100 days (twice 
the period of distraction after lengthening to consolidate the regenerate). Accordion manoeuvre (AM) at fracture site was started as per Baruah \& Patowary protocol [2] once patient became comfortable with apparatus and started mobilization with support. Distraction was done first at the rate of $0.25 \mathrm{~mm}$ twice a day for 7 days followed by rest for 3-4 days. Then, compression was done in the same rate followed by rest. These manoeuvres constituted a cycle and each cycle was performed twice. After two cycles of AM, final compression was done at the rate of $0.25 \mathrm{~mm}$ on every 3rd day for 1 month.

Initially shoe raise was provided by rubberized sandal attached to the foot to compensate for shortening so that the patient could bear weight as well. Patient was discharged from the hospital when he became well versed with care of the apparatus and could perform AM himself. He attended OPD every month till fracture united as confirmed clinically and radiologically. The apparatus was dynamized for one month and removed at 6 th month.

\section{Results}

Patient was followed up regularly till final check-up at about 10 years, when he visited OPD himself to showcase his satisfaction with treatment. He regained normal length $\&$ full function of the affected limb (Fig. 1). There was no residual rotational deformity. There was no major complication except superficial pin tract infections which were treated with dressings. He could go back to his pre-injury working status.

\section{Discussion}

The management of acute fractures is delayed for different reasons in developing countries like India. Various authors had coined them as Neglected fractures. However, nobody has tried to define the period of delay to coin such a fracture as "neglected" [1].

The term "Postponed Fractures" seems to be logical in such cases that keeps aside the derogatory term Neglect that has extreme connotations. In PFs, because of delay, proper management according to standard protocol for fresh fractures cannot be offered and, hence, newer concept emerged called "Fixation in situ" (FIS).

The postponement of management in fractures is a common scenario in India due to four Ps namely Poor Finance, Poor transport and communication, Poor nutrition, Paucity of operation theatre (OT) days. Since the treating doctors have no control on these factors, attempt to discover newer concepts with available technology becomes rational.
Three components of the Ilizarov technology, namely Ring Fixator, Corticotomy and Accordion Maneuver, were utilized to get our proposed results. All these components work in harmony based on the sound biologic law of tissue genesis and growth known as "Law of Tension Stress" discovered by Ilizarov [3].

Ring assembly was the first component used to provide stability to the construct. This external fixator provided optimum biologic and mechanical conditions for regeneration, remodeling and rehabilitation in the treatment of fractures and Orthopaedic diseases [3] Moreover, principle of biological fixation without unnecessary soft tissue handling could be applied through this external fixator.

Corticotomy was another principle that provided the basic requirement to lengthen and or to de-rotate rotated fragments. It was the basis for subsequent neo-histogenesis. It was a refined and complex surgical procedure that was developed by Ilizarov in 1960s [4].

The capability of correcting rotational deformity is one of the important tools in the armamentorium of Ilizarov. It is generally advised that rotation is to be corrected at the end of distraction gradually. This is usually achieved by "Rotation correction devices" being mounted with supports and posts along with threaded rods. If de-rotation is to be combined with lengthening, it is advised to start derotating only after 2-3 weeks of lengthening when bone regeneration becomes evident in X-ray or Sonography. However, if the rotational deformity does not exceed 10-15 degrees, an acute correction can be done by a special maneuver of staggering rods [5]. Our patient had 15 degrees of External rotation deformity at the fracture site. It appeared to be more than that because of postural external rotation at the hip joint. We corrected the 15 degrees of deformity acutely on table by the above technique in our case.

Lengthening was done after 7 days of latency at the rate of $0.25 \mathrm{~mm}$ in four sittings. After length was regained, the apparatus was maintained for a period twice the period taken to lengthen to get satisfactory consolidation of the regenerate. Patient was encouraged to bear weight on the affected limb to enhance formation of healthy regenerate and subsequent consolidation. Retensioning of wires and Tightening of wire clamp and Schanz pin clamps were mandatory in each visit. Meticulous Pintract care was advised to reduce pin tract infections. He was advised to take a regular bath.

Accordion maneuver was used to stimulate healing at the fracture site. Accordion Maneuver (AM) is the "Bloodless stimulation" of bone healing as described by Professor Ilizarov. It comprised alternate compression and distraction which converted the scar tissue at the fracture non-union site into tissues capable of neo-osteogenesis [6]. Apart from non-union, a modified form of this tool can be used in hyporegenerate developed in bone transport and at docking site after acute docking or after internal bone transport [7]. There 


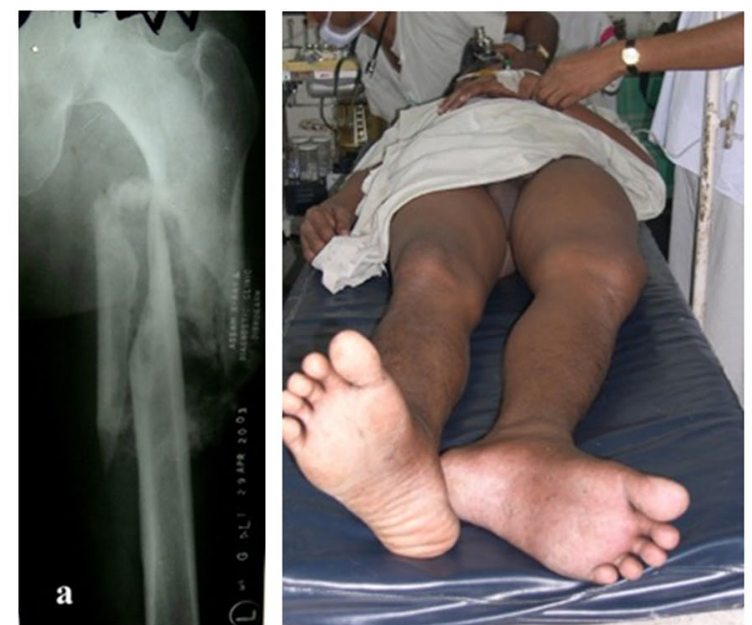

(a)

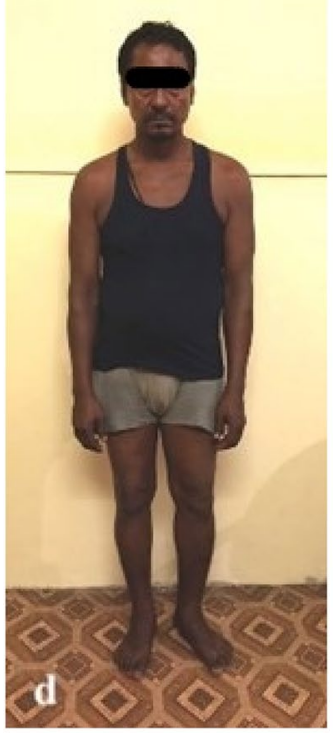

(d)

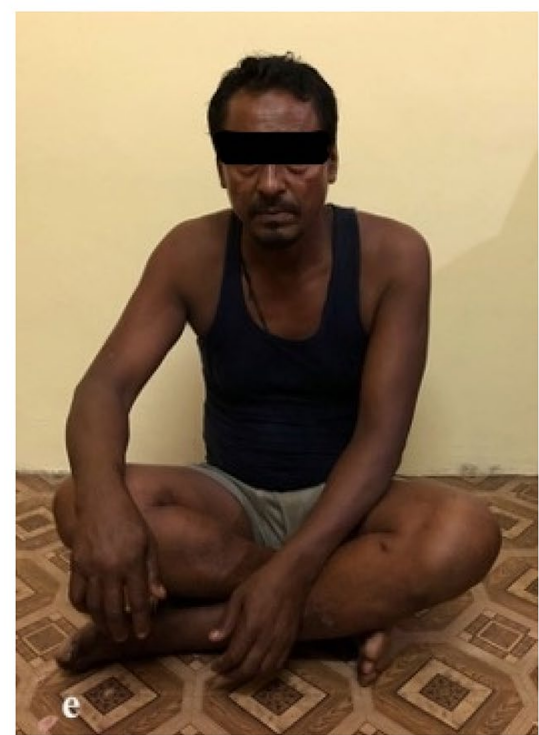

(e)

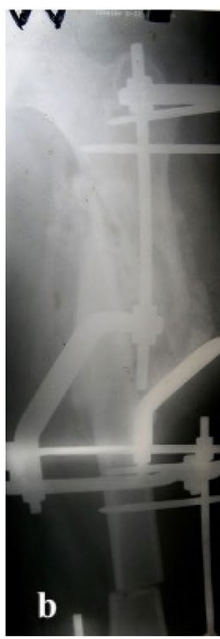

(b)

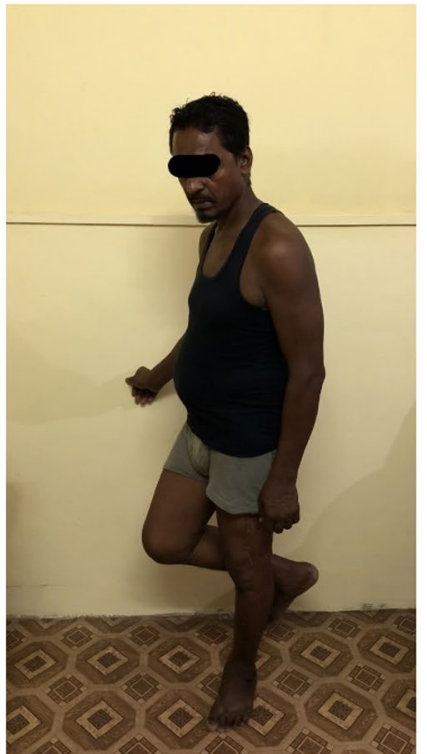

(f)

Fig. 1 a Pre-op X-ray and clinical picture of patient. b X-ray during Treatment Process. c Follow-up X-ray showing radiological union. d-f Clinical images at final follow up

is a paucity of literature explaining the protocol and rationale for use of AM in various conditions. We used the Protocol of AM as per Baruah \& Patowary. We feel AM, a bloodless tool, hastened this process. It is needless to emphasise that we developed sufficient confidence on AM, being used in another 23 cases to this effect [2]. AM was done in our patient at fracture site only. This maneuver acted like axial micromotion to enhance healing so that the apparatus could be removed at 6th month after period of distraction and consolidation.
We could achieve healing of the fracture without trying to reduce by open method but by dealing the fracture site indirectly by fixing it in situ. Corticotomy that was done far distal to fracture site helped to correct external rotation deformity and shortening. It is true that Ilizarov concept and technology had revolutionized the Orthopaedic treatment. It forms bone without bone grafting and facilitates soft tissue closure without plastic reconstruction [8]. The uniqueness of our case is that we have performed rotation and length correction at different site rather than fracture site 
and we could not find any mention of this concept of "Fixation in situ" in the available English literature. Therefore, this becomes a novel concept and can benefit selected patient by its application.

The principle followed commonly in majority of acute diaphyseal fractures of long bones is to attain optimal reduction in terms of alignment, rotation and length and provide stability. In spite of being postponed, we could achieve satisfactory functional and radiological result in our case nearly fulfilling the aforesaid principle.

Since the patient lost the 10 years of follow-up X-rays in flood and because of COVID-19 pandemic being severe, we could not call back the patient for repeat X-ray which we consider as a minor limitation in this paper.

\section{Conclusion}

Fixation in situ is a novel technique to treat postponed fracture of diaphysis of femur by Ilizarov. It yields satisfactory functional results when combined with stability, Corticotomy and AM. With one case, it is not possible to render a concrete conclusion. However, it can be an alternative solution in specific difficult situation.

\section{Compliance with Ethical Standards}

Conflicts of interest There are no conflicts of interest.

Declaration of patient consent The authors certify that they have obtained all appropriate patient consent forms. In the form, the patient has given consent for his images and other clinical information to be reported in the journal.

\section{References}

1. Meyers, M. H., Harvey, J., \& Moore, T. M. (1973). Treatment of displaced subcapital and transcervical fractures of the femoral neck by muscle-pedicle-bone graft and internal fixation. A preliminary report on one hundred and fifty cases. J Bone Joint Surg Am., 55, 257-274.

2. Baruah, R. K., \& Patowary, S. (2018). Accordian Manoeuvre: A bloodless tool in Ilizarov. J Limb Lengthen Reconstr., 4(1), 11-19.

3. Ilizarov GA. Transosseous Osteosynthesis, 1st ed. Green SA, editor. Berlin, Springer -Verlag; 2000.

4. Golyakhovsky V, Frankel VH. Ilizarov Corticotomy (Compactotomy) Techniques. In Golyakhovsky V, Frankel VH, editors. Text Book of Ilizarov Surgical Techniques. Bone correction and Lengthening. 1st ed. New Delhi: Jaypee Brothers Medical Publishers (P) Ltd; 2010; p. 126-141.

5. Golyakhovsky V, Frankel VH. Hinges. In Golyakhovsky V, Frankel VH, editors. Text Book of Ilizarov Surgical Techniques. Bone correction and Lengthening. 1st ed. New Delhi: Jaypee Brothers Medical Publishers (P) Ltd; 2010; p. 156-158.

6. Golyakhovsky V, Frankel VH. General Principles of Ilizarov Technique. In Golyakhovsky V, Frankel VH, editors. Text Book of Ilizarov Surgical Techniques. Bone correction and Lengthening. 1st ed. New Delhi: Jaypee Brothers Medical Publishers (P) Ltd; 2010; p. 168

7. Paley D. Problems, Obstacles and complications of limb lengthening. In: Operative Principles of Ilizarov By ASAMI group. Baltimore,Maryland, USA: Wiliams \& Wilkins; 1991. p. 352-365.

8. Cattaneo, R., Catagni, M., \& Johnson, E. (1992). The treatment of infected non unions and segmental defects of the tibia by the methods of Ilizarov. Clin Orthop Relat Res., 280, 143-52.

Publisher's Note Springer Nature remains neutral with regard to jurisdictional claims in published maps and institutional affiliations. 